\title{
FUNDAMENTAL STUDIES OF FLUID MECHANICS: STABILTY IN POROUS MEDIA
}

\author{
DE-FG02-05ER15692
}

FINAL REPORT

\author{
G. M. Homsy \\ Professor Emeritus \\ Department of Mechanical and Environmental Engineering \\ University of California at Santa Barbara \\ Santa Barbara, CA 93106-5070 \\ 805-636-9366 \\ bud@engr.ucsb.edu
}

February 2014 


\section{Introduction}

This is the final technical report for our grant "Fundamental Studies of Fluid Mechanics: Stability in Porous Media”, DE-FG02-05ER15692. Our work has been concerned with theoretical, computational and experimental studies of a variety of flow and transport problems of generic interest and applicability in energy-related and energy-intensive processes. Research during the grant period has focused on three main areas:

(i) Coating flows: these are of interest in information technologies, including the manufacture of integrated circuits and data storage and retrieval devices. It is estimated that $50-70 \%$ of the starting raw materials and intermediate devices in information technology processes must be discarded as a result of imperfections and failure to meet specifications. These in turn are often the result of the inability to control fluid-mechanical processes and flow instabilities.

(ii) Dispersed multiphase flow: these are of interest in separation processes, direct contact heat and mass transfer, and mixing under conditions of slow Stokes flows. Efficient mixing and transport of energy is a challenge for small scale systems.

(iii) Wetting and spreading: these are of interest in microfluidic applications, as well as in phase change devices such as heat pipes and thin film evaporators.

Our work over the grant period is primarily fundamental in nature. We are interested in establishing general principles and behaviors that relate to a variety of processes in a variety of contexts. Our work has focused on fluid mechanical phenomena that are of interest in energyrelated technologies, with an emphasis on interfacial flows.

\section{Activities}

This progress report covers the period, August 1, 2005 through July 31, 2009. Only the main achievements are summarized. More detail is contained in the various journal articles published during the last grant period, which are referenced below.

\section{Students Supervised}

We have worked with a number of graduate students, postdoctoral fellows, and collaborators.

\section{PhD Students}

Dr. Thomas Ward - Dr. Ward completed his PhD in Fall, 2003 and is currently on the faculty at Iowa State University.

Dr. Xiu Mei Xu - Dr. Xu completed her PhD in Summer, 2009 and is currently a research staff scientist at the IMEC in Belgium.

\section{Postdoctoral Fellows}

Dr. Juan Fernandez - Dr. Fernandez received his PhD in Physics at ESPCI, Paris. He is currently a Professor in Sevilla, Spain. 
Dr. Rouslan Krechetnikov - Dr. Krechetnikov received his $\mathrm{PhD}$ in Mechanical Engineering at Univ. of Notre Dame. He is currently an Associate Professor of Mathematics at the Univ. Alberta, Edmonton.

Dr. Etienne Lac - Dr. Lac received his PhD at the Univ. Compiegne, France. He is currently with Schlumberger Corporation, UK.

Dr. Malika Ouriemi - Dr. Ouriemi received her PhD from Univ. Marseille, France. She is currently with the Institut de Francais Petrole, Lyon, France.

\section{Collaborators}

Dr. Volodia Ajaev - Dr. Ajaev collaborated with the PI in writing an invited review paper in the Ann. Rev. of Fluid Mech. He is Professor of Mathematics at SMU, Dallas TX.

Dr. Juan Gomba - Dr. Gomba was a Fullbright Fellow and worked with us over the period of one year. He is currently a professor at the Universidad Nacional del Centro de la Provincia de Buenos Aires, Argentina

Dr.Christo Christov - The late Dr. Christov was a Professor of Mathematics at Louisiana State University.

Dr. Eric Lajeunesse - Dr. Lajeunesse received his PhD from Universite Paris Sud in 1998. He is currently Professor of Geophysics at Universite Paris VI, France.

\section{Publications}

Below is a list of papers published on work supported by this grant (2005-present)

\section{Papers in Print}

1. Thomas Ward \& G. M. Homsy, "Chaotic streamlines in a translating drop with a uniform electric field", with Thomas Ward, J. Fluid Mech. 547,215 (2006).

2. Rouslan Krechetnikov \& G. M. Homsy, "Dip coating in the presence of a substrate-liquid interaction potential”, Phys. Fluids 17, 102105 (2005).

3. E. Lajeunesse, J-B Monnier, \& G. M. Homsy "Granular slumping on a horizontal surface", Phys. Fluids, 17, 103302 (2005).

4. Rouslan Krechetnikov \& G. M. Homsy, "Surfactant effects in the Landau-Levich problem", J. Fluid Mech. 559, 429 (2006).

5. Xiu Mei Xu \& G. M. Homsy, "“The settling velocity and shape deformation of drops in a uniform electric field", J. Fluid Mech. 562, 445 (2006).

6. V. Ajaev \& G. M. Homsy, "Modeling shapes and dynamics of confined bubbles", Annual

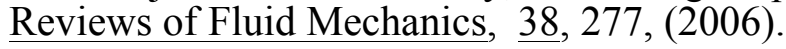

7. Rouslan Krechetnikov \& G. M. Homsy, "Experimental study of substrate roughness and surfactant effects on the Landau-Levich law", Phys. Fluids, 17, 102108 (2006). 
8. Xiu Mei Xu \& G. M. Homsy, "Three-dimensional chaotic mixing inside drops driven by a transient electric field", Phys. Fluids 19, 13102 (2007).

9. E. Lac \& G. M. Homsy, "Axisymmetric deformation and stability of a viscous drop in a steady electric field”, J. Fluid Mech. 590, 239 (2007).

10. J. M. Gomba \& G. M. Homsy, “Analytical solutions for partially wetting two dimensional droplets”, Langmuir, 25, 5684 (2009).

11. Christo Christov \& G. M. Homsy, "Enhancement of transport from drops by modulated electric fields", Phys. Fluids 21, 83102 (2009).

12. J. M. Gomba \& G. M. Homsy, "Regimes of thermocapillary migration of droplets under partial wetting conditions", J. Fluid Mech. 647, 125 (2010).

13. Malika Ouriemi \& G. M. Homsy, "Experimental study of the effect of surface-absorbed hydrophobic particles on the Landau-Levich law", Phys. Fluids 25, 82111, (2013).

Papers in Press

None

\section{Invited Talks, Seminars, and Presentations}

Major Invited Talks and Plenary Lectures - G. M. Homsy

Invited talk at International Chemical Engineering Congress, Mumbai, India. Jan. 2006.

Invited talk at the European Coating Symposium, Paris, France. Sept. 2007.

Distinguished Lecturer, College of Engineering, Univ. Tennessee, Knoxville, TN. Nov. 2008.

Invited talk at International Congress on Heat Transfer, Namur, Belgium. May, 2009.

\section{Other Presentations}

The Principal Investigator and his students have given many other presentations of DOEsponsored work over the grant period. These include departmental seminars by the PI at UCLA, UCSD, Univ. British Columbia, Imperial College, Los Alamos National Lab, UC Riverside, Paris VI, Univ. Marseille, Univ. Toulouse, Stanford, Univ. Maryland, Washington Univ., Princeton, Brown Univ. Georgia Tech, Univ. New Mexico, and over 20 contributed papers at annual meetings of the AIChE and the Division of Fluid Dynamics of the APS. These will not be listed separately in the interests of brevity.

\section{Research Summaries}

Here we give summaries of the results obtained over the last grant period. Citations in square brackets refer to the list of papers given above. Our work on granular slumping [3] is not described here. Full details of all the work are given in the papers themselves.

\section{Coating Flows}

Coating flows pertain to the subject of the flow and adhesion of liquids to solid substrates. Coating theory and practice seek to predict and control the thickness of the liquid that is applied. A significant body of knowledge exists for the coating of Newtonian liquids with clean interfaces over mathematically smooth substrates. Since it is rare if at all that coating takes place under 
such circumstances, our work has focused on the effects of (i) topography, (ii) an interaction potentials between liquid and solid, (ii) Marangoni stresses due to surfactants, and (iii) small adsorbed particles.

In [2] we investigate the Landau-Levich problem of dip coating in the presence of a strong interaction potential normal to the substrate. This study is motivated by coating at very low capillary numbers when the deposited film thickness is less than $1 \mu \mathrm{m}$ and such interaction forces may become important. In the presence of an extra body force the solution procedure differs significantly from the classical one and leads to substantial deviations from the Landau-Levich law for the entrained film thickness. In particular, attractive potentials produce film thickening and the resulting film thickness is independent of speed to lowest order. Repulsive potentials bring about more complicated behavior and lead either to films whose thickness is also independent of speed, or to a modification of the leading order constant in the classical Landau Levich $C a^{2 / 3}$ law, where $C a$ is the capillary number.

Coating of complex fluids over rough substrates is of great technological interest. In [4]we investigate the coating of liquids containing a soluble surfactant. Our main observations are that pure hydrodynamical modelling of surfactant effects with simple inflow and outflow boundary conditions predicts film thinning, where experiments by us [7] and others show film thickening. We conclude that such simple boundary conditions and models of material behavior may not be sufficient to explain the film thickening observed in many experiments. In addition to these experiments using surfactants, in [7] we also examine the effect of well-characterized substrate roughness on the coated film thickness, which reveals its influence on wetting-related processes as an effective boundary condition at the wall. In particular, roughness results in a significant thickening of the film relative to that on a smooth substrate and a different power of capillary number than the classical Landau-Levich law.

In a further study of the effect of complex systems on coating [13], we consider dip coating of liquids with particles absorbed on the interface under controlled conditions and measure the coating thickness of particle dispersions as a function of coating speed, physical properties, and particle surface concentration. The thickness of the coating film is measured over a wide range of capillary numbers for two different kinds of particles. It is found that the presence of particles results in a significant thickening of the film relative to that for a clean interface, and in a slightly different power law for the thickness vs. capillary number than the classical Landau-Levich law. The influence of the particle surface concentration is also investigated, and shows a strong dependence of the film thickness on concentration, including a threshold concentration at which the film thickness begins to deviate from that for a clean interface.

\section{Electrohydrodynamics of Drops}

Determining the steady shape of drops subject to electrical stresses is one of the most fundamental problems in the field. In [9], we consider a neutrally buoyant drop in a second liquid subjected to a uniform electric field, with both liquids taken to be leaky dielectrics. The jump in electrical properties creates an electric stress balanced by hydrodynamic and capillary stresses. The electric and flow fields are determined numerically using boundary integral techniques. The drop deforms into a prolate or an oblate spheroid, depending on the values of property ratios ratios $R$ (resistivities), $Q$ (permitivities) and $\lambda$ (dynamic viscosities). The ratio of 
the electric stress to the drop interfacial tension is given by the dimensionless electric capillary number, $C a_{e}$. For $\lambda=1$, we present a survey of the various behaviours obtained for a wide range of $R$ and $Q$. We delineate regions in the $(R, Q)$ plane in which the drop either attains a steady shape under any field strength or reaches a fold-point instability past a critical $C a_{e}$. Various break-up modes are identified, as well as more complex behaviours such as bifurcations and transition from unstable to stable solution branches. We also show how the viscosity contrast can stabilize the drop or advance break-up in the different situations encountered for $\lambda=1$.

Settling of drops in electric fields is also of interest. In [5], we theoretically and experimentally investigate the settling velocity and deformation of a leaky dielectric liquid drop in a second leaky dielectric liquid subject to a uniform electric field, $E$. Both shape distortion and charge convection, when coupled with the asymmetric velocity profiles, will produce a net drag and a shift in the settling speed. Perturbation methods for small shape distortion and small charge convection are used to solve for this shift, which predict a dependence on the drop size. Experiments are performed to measure the settling velocity and deformation of phenylmethylsiloxane-dimethylsiloxane (PMM) drops in castor oil. The experimental results are in qualitative agreement with the theory: the symmetric and asymmetric deformations and the change in settling velocity are all proportional to $E^{2}$ as predicted, and the settling speed shows the correct trends with drop size.

Mixing of fluids can be very slow in stagnant liquids and low Reynolds number flows. In addition, the transport of heat or mass can be similarly slow if the flow structure contains significant regions of closed steamline patterns, regardless of the Peclet number. The reason is that the net transport rate is limited by transport across streamlines which, in a steady flow, is diffusion limited. Chaotic advection has been generally recognized as an attractive way to mix fluids that would otherwise would be very difficult to mix (because of the high fluid viscosity, low velocity, or small scale size of the flow.)

In [1], we consider a drop translating in the presence of a uniform electric field from both theoretical and experimental points of view in order to determine qualitative properties of threedimensional chaotic particle trajectories and mixing in bounded Stokes flows. The flow is a combination of a Hadamard-Rybczynski and a Taylor circulation due to the translation and electric field respectively. The three-dimensional trajectories are generated by tilting the electric field relative to the drop translational motion by an angle $\alpha$. The numerical analysis includes qualitative analysis of the degree of mixing by Poincaré mapping, quantitative estimates of the largest mixed volume fraction and the rate of mixing characterized by the largest Lyapunov exponent. Experiments are performed using a castor oil/silicone oil system for the continuous and dispersed phases respectively. Single trajectories are studied by visualizing small neutrally buoyant glass particles inside the dispersed phase using a stereoscopic particle tracking technique. We observe crossings of the unperturbed separatrix and particle trajectories that show evidence of a symmetry plane, both important features of the theory.

Settling is not the only mechanism for producing mixing. In [8], we numerically study the threedimensional (3D) chaotic trajectories inside a neutrally buoyant drop driven by periodically switching a uniform electric field through an angle $\alpha_{\mathrm{m}}$. Periodically switching the field alternates the direction of the interfacial electric force; thus, symmetries of the steady Taylor circulation are 
broken and 3D chaotic advection is generated inside the drop. The extent of the chaotic mixing is related to two parameters: the switching angle $\alpha_{\mathrm{m}}$ and modulation period T. The parametric dependence of mixing is investigated by tracing trajectories of Lagrangian particles. Analysis shows that depending on the range of $\alpha_{\mathrm{m}}$ there are either four or eight center fixed points (CFPs), around which invariant surfaces exist and chaotic mixing is suppressed. Our calculations of the mixed volume fraction and Lyapunov exponents suggest an optimal range of $\alpha_{\mathrm{m}}$ for best mixing. For a given $\alpha_{\mathrm{m}}$, by varying modulation period T, we find that a resonant phenomena may occur, and global uniform mixing is possible.

Transport of heat or mass from circulating droplets that are both settling and subject to an axial electric field is of fundamental interest. The electric field can be either steady or oscillatory in time and drives an electrohydrodynamic flow, called the Taylor circulation, which augments the Hadamard circulation caused by steady translation. The problem is governed by four dimensionless groups: the Peclet number Pe, the dimensionless amplitudes of both the steady and unsteady electric field, and the dimensionless frequency $\omega$ of the modulation. In [11], the convective diffusion equation is solved numerically over a wide range of parameters - in particular, very large Peclet numbers. The results are characterized by the asymptotic rate of extraction of heat or mass from the droplet, which is found to be exponential in time. The enhancement factor, defined as the ratio of this rate to that of a stagnant drop, is studied as a function of parameters. For steady drops, we find that transport remains diffusion controlled, but the enhancement factor is significantly higher with the Taylor flow than without. For modulated electric field the enhancement factor is not a simple function of parameters and exhibits spectral "resonant peaks" at particular values of $\omega$ for which the enhancement factor is extremely large. Movies of the simulations are used to study the underlying time-periodic spatial structures of the concentration field (so-called strange eigenmodes) and the complex time dependence that is responsible for these resonances.

Wetting and Spreading

Manipulation of small liquid droplets on solid substrates is key to the success of many microfluidics applications. Causing liquids to migrate under partially wetting conditions is also a key mechanism in many processes for migration, banking, and production of oil in mixed wettability reservoirs.

The first step in understanding such problems is to describe the static droplet shape. In [10], we present a new analytical solution for the static shape of a droplet in equilibrium with a surrounding thin film on a solid substrate. The modeling includes the effects of capillarity and disjoining-conjoining pressure accounting for intermolecular forces between the solid and the liquid. We derive new analytical solutions for the shape of the droplet, the cross-sectional area, the half-width, and the maximum curvature and inflection points, and we study the effects of the size of the droplet on the apparent contact angle.

We then exploit this modeling to study the migration of droplets due to surface tension gradients by modeling the thermocapillary migration of two-dimensional droplets of partially wetting liquids on a non-uniform heated substrate [12]. The model includes the effect of a non-zero contact angle introduced through a disjoining-conjoining pressure term. We allow the droplet to change its profile with time and identify and describe three different regimes of behaviour. For small contact angles, the droplet spreads into a long film profile with a capillary ridge near the leading edge, a behaviour that resembles the experiments on Marangoni films. For large contact 
angles, the droplet moves as a single entity, weakly distorted from its static shape, as in most experiments on thermocapillary migration of droplets. We also show some intriguing morphologies that appear in the transition between these two regimes, including secondary droplet formation and stranding.

\section{Major Review Article}

Much of our research supported by previous DOE grants (the current one of which is an extension), focused on a variety of problems involving drops and bubbles in confined spaces. These types of problems arise in pore-scale modeling of oil recovery as well as in microfluidics applications. With Prof. Ajaev, (a former postdoctoral fellow), we co-authored a major review article in Ann. Rev. Fluid Mech. [6]. Reviews in this prestigious series are by invitation only.

In [6], we review mathematical models of confined bubbles, emphasizing physical mechanisms as expressed in simple geometries. Molecular interactions between liquid, gas, and the confining solid are all-important and are described through the disjoining pressure concept. Methods for finding static shapes are considered. The static solution is a springboard for discussing pressuredriven and surface-tension-driven flows, both of which involve viscous effects and macroscopic films entrained near apparent contact lines. We next discuss vapor bubbles produced by thermal effects. Vaporization localized near contact lines and condensation distributed in colder parts of the interface lead to steady vapor bubbles. Their size is determined through global constraints. Unsteady vapor bubbles are discussed and we end with thoughts on open problems. 